\title{
Hemosiderin in Decidua Basalis
}

National Cancer Institute

\section{Source}

National Cancer Institute. Hemosiderin in Decidua Basalis. NCI Thesaurus. Code

C118150.

The deposition of hemosiderin along the gravid uterine fundus beneath the placenta. 